\title{
Collections universitaires et histoire des sciences : étude d'un dispositif pour la formation professionnelle des enseignants
}

\section{Muriel Guedj}

\section{OpenEdition}

\section{Journals}

Édition électronique

URL : http://journals.openedition.org/trema/3904

DOI : 10.4000/trema.3904

ISSN : 2107-0997

Éditeur

Faculté d'Éducation de l'université de Montpellier

Édition imprimée

Date de publication : 1 mars 2018

Pagination : $51-74$

ISBN : 979-10-96627-04-2

ISSN : 1167-315X

\section{Référence électronique}

Muriel Guedj, « Collections universitaires et histoire des sciences : étude d'un dispositif pour la

formation professionnelle des enseignants », Tréma [En ligne], 48 | 2018, mis en ligne le 01 juin 2018, consulté le 21 avril 2019. URL : http://journals.openedition.org/trema/3904 ; DOI : 10.4000/

trema.3904

Ce document a été généré automatiquement le 21 avril 2019

Trema 


\title{
Collections universitaires et histoire des sciences : étude d'un dispositif pour la formation professionnelle des enseignants
}

\author{
Muriel Guedj
}

\section{Introduction}

1 Si l'Epistémologie, l'Histoire des Sciences et des Techniques (EHST) et la formation professionnelle sont deux domaines trop rarement associés, les travaux ${ }^{1}$ qui s'emparent de cette question s'accordent pour reconnaître l'EHST comme étant un domaine particulièrement fécond pour l'expression de la professionnalité, que celle-ci concerne la formation des ingénieurs, des médecins ou des enseignants. L'étude de cas détaillée ici s'inscrit dans cette dynamique avec la proposition d'une formation, conjuguant EHST et formation professionnelle ; le public visé étant constitué de futurs enseignants.

Le choix est fait pour cette formation d'introduire l'EHST à partir d'objets scientifiques issus des collections universitaires car, outre la volonté de faire découvrir le patrimoine de l'université ${ }^{2}$ la dimension matérielle, par les approches spécifiques qu'elle convoque, facilite l'introduction à une histoire sociale et culturelle. Cependant la formation n'est pas entièrement dédiée à l'EHST et l'entrée historique est l'occasion d'une structuration d'ensemble permettant de développer autour de projets collaboratifs, des compétences professionnelles reconnues comme étant attachées à la pédagogie de projet. En montrant que ces compétences professionnelles relèvent également de l'EHST, l'étude de cas présentée constitue une illustration originale à la complémentarité des approches association de la pédagogie de projet et l'EHST - et contribue à renforcer le lien entre EHST et professionnalité.

3 Cet article se structure selon trois parties. La première partie consiste à présenter en détail le contexte associé au dispositif mis en place pour la formation alors que la seconde 
partie s'attache à décrire et justifier les choix opérés pour cette formation. La troisième et dernière partie consiste en une analyse de la formation eu égard aux compétences professionnelles visées pour cette formation.

\section{Un contexte singulier}

\section{1. L'Unité d'Enseignement « Projet »}

4 L'étude de cas présentée dans ce travail concerne la formation professionnelle d'enseignants du premier degré $e^{3}$ au sein des masters MEEF (Métiers Enseignement Education et Formation) qui structurent la formation des futurs enseignants et des personnels de l'éducation. Elle s'intéresse à la conception et la mise en place d'une unité d'enseignement (UE) relative à la pédagogie de projet; s'inscrivant ainsi dans les dynamiques qui questionnent les liens entre recherche et formation au sein des Écoles Supérieures du Professorat et de l'Education (ESPE).

5 En réponse à une demande institutionnelle, l'UE vise à impliquer les étudiants pour concevoir et développer des dispositifs relevant de la pédagogie de projet et être en mesure de conduire une réflexion critique relativement aux apprentissages développés dans cette formation.

\section{2. L'exposition Regards croisés sur la lumière : un terrain expérimental}

6 L'installation d'une exposition intitulée "Regards croisés sur la lumière ${ }^{4}$ au sein de l'université de Montpellier est l'occasion de structurer cette UE en offrant un terrain « expérimental » aux étudiants permettant à ces derniers de conduire un projet qui leur est propre.

7 Dans le cadre de l'année internationale de la lumière ${ }^{5}$, l'exposition propose de faire découvrir les grandes problématiques de la lumière en croisant approches historiques et scientifiques. Ainsi la question concernant la nature de la lumière se structure autour de phénomènes optiques présents dans la nature (la réfraction, la diffraction, la réflexion, aurores boréales, coucher/lever de Soleil etc.) alors que la captation de la lumière constitue un second thème à partir duquel s'articulent la vision (celle de l'homme, celle des animaux nocturnes) et la vision augmentée (avec l'usage des instruments). Une thématique dédiée à l'articulation entre lumière et image (appareil photographique etc.) vient compléter cet ensemble.

Les objets présentés sont tous issus des collections universitaires; c'est également le cas des ouvrages sélectionnés qui appartiennent au service du patrimoine écrit et graphique de l'université (SPEG).

Le terme d'objet inclut les instruments scientifiques, les artefacts en usage dans le champ de l'anthropologie, les témoins exemplaires pour les techniques ou encore les spécimens des sciences naturelles. Cette multiplicité de termes conduit Serge Chaumier ${ }^{6}$ à un travail de définition qu'il conclut par la généralisation du terme objet quel que soit le domaine concerné. En référence à son travail, le terme d'objet est retenu pour cette étude.

Des ateliers scientifiques enrichissent l'exposition. Ces ateliers visent à présenter plus en détail certains des phénomènes scientifiques rencontrés tout au long du parcours muséal. 
Ainsi, l'utilisation d'un instrument, la modélisation d'un phénomène ou l'approche expérimentale permettent de questionner les phénomènes, d'en repérer les spécificités et d'inscrire leurs découvertes dans des contextes situés historiquement. Cette approche a semblé essentielle pour "aiguiser » le regard du visiteur. Les ateliers sont conduits par des doctorants et des enseignants chercheurs scientifiques pour lesquels un travail de médiation préalable a permis d'harmoniser les présentations.

\section{3. Le guide du visiteur}

11 La volonté d'impliquer les étudiants du master MEEF, futurs enseignants, a clairement orienté les concepteurs de l'exposition à privilégier le public scolaire - primaire et secondaire - sans toutefois que ce choix ne soit exclusif. C'est dans ce contexte que les étudiants devront concevoir et réaliser un document permettant à un enseignant d'organiser une visite originale avec une classe. Ce $"$ guide du visiteur» ${ }^{7}$ qui doit proposer un parcours thématique dans l'exposition ainsi que des activités à réaliser avec les élèves, est élaboré à partir d'un cahier des charges et selon des modalités qui sont détaillées ciaprès.

Regards croisés sur la lumière

Cahier des charges pour un Guide du visiteur

Présentation de l'exposition dans son ensemble

- Situer l'exposition (constitution, destination, inscription nationale);

- Renseigner succinctement l'ensemble des thèmes présentés dans l'exposition.

Proposition d'un parcours spécifique

- Choisir quelques objets (4 objets au maximum) des collections ;

- Documenter ces objets avec des éléments historiques spécifiques : types d'usages (enseignement, recherche, etc.), usagers (enseignants, chercheurs, techniciens) fabricants, lieux d'usages (institutions concernées), disciplines concernées, phénomènes scientifiques associés etc ;

- Proposer un parcours spécifique dans l'exposition à partir de ces objets.

- Choisir un phénomène scientifique en lien avec les objets, le décrire et expliciter les concepts scientifiques sous-jacents avec un niveau de formulation adapté aux enseignants du premier degré. Proposer une activité scientifique destinée à un public scolaire permettant une première approche du phénomène. Associer cette activité au parcours.

- Prévoir des activités pour le visiteur (jeux, quizz, expériences etc.) ;

- Proposer une rubrique pour « aller plus loin».

Original et ludique, rigoureux quant aux informations, le Guide du visiteur peut prendre la forme d'un support « papier » ou numérique (smartphone, tablette).

Format à adapter au parcours (difficulté, durée envisagée, public visé etc.).

\section{Le dispositif de formation}

Cette partie vise à présenter le dispositif de formation en explicitant les étapes qui la composent puis en détaillant les objectifs visés. Elle s'achève avec la présentation de l'élaboration du dispositif muséal, projet plus vaste dans lequel la formation s'inscrit. Ce dernier aspect est essentiel pour saisir le rôle joué par les documents mis à disposition des étudiants. 


\section{1. Présentation d'ensemble}

13 La première étape de la formation consiste en une visite commentée de l'exposition qui permet aux étudiants de découvrir les objets, les documents patrimoniaux et les ateliers scientifiques relatifs à chaque thématique.

Alors que les objets des collections sont accessibles de manière occasionnelle (il n'existe pas de musée dédié) et que le patrimoine graphique reste largement méconnu, cette visite constitue pour une large majorité d'étudiants une première rencontre avec le patrimoine de l'université. Indépendamment de cette découverte, la visite a pour objectif de montrer l'intérêt d'associer aux objets des collections ceux du patrimoine écrit, afin de pouvoir situer les objets dans des contextes historiques, scientifiques et culturels. La mise en évidence des usages spécifiques aux objets contribue à ce travail de contextualisation. Qu'il s'agisse de pratiques d'enseignement ou de recherche, ces objets sont les témoins d'une histoire scientifique locale que cette approche contribue à éclairer.

La seconde étape réside dans la présentation d'un cahier des charges ${ }^{8}$ afin d'indiquer les objectifs visés et préciser les modalités du travail à réaliser.

Comme cela a déjà été évoqué, il s'agit pour les étudiants de construire un parcours thématique au sein de l'exposition devant permettre à un enseignant de conduire une visite pour une classe. Pour cela un travail spécifique doit être effectué autour de quelques objets présentés dans l'exposition. Ainsi, 3 ou 4 objets des collections ou du patrimoine graphique doivent être sélectionnés puis documentés à partir d'un travail historique. Quels sont les usages associés à ces objets et pour quelles finalités ? Qui sont les usagers et quelles pratiques sont associées à ces objets? Quelles évolutions concernant les pratiques, les usagers et les objets eux-mêmes peut-on lire? Qui fabrique l'objet et dans quel contexte; est-ce une commande, un objet standard etc.? Les documents patrimoniaux sont également à expliciter ; pour chacun d'eux, auteur, date et contenus sont à préciser et à mettre en lien avec les objets sélectionnés. Qu'apportent-ils aux contextes historique et scientifique d'ensemble?

17 Le choix des objets définit ainsi le thème du parcours.

18 Par ailleurs, afin que chaque visite soit l'occasion de mettre l'accent sur une dimension scientifique associée à la thématique retenue, le cahier des charges invite les étudiants à sélectionner un phénomène scientifique. Celui-ci doit doit être explicité et donner lieu, au sein du parcours, à des propositions d'activités destinées aux élèves.

19 C'est à partir de ces indications fixées par le cahier des charges que le guide du visiteur doit être conçu. Indépendamment de ce texte de cadrage, les étudiants disposent d'un document qui présente, pour chaque objet, une description succincte et une photographie constituant un récapitulatif des thèmes abordés offrant ainsi un regard d'ensemble sur l'exposition. Élaboré par les concepteurs de l'exposition ${ }^{9}$ ce document, qualifié de pilote permet au lecteur qui n'a pas eu l'occasion de visiter l'exposition d'en découvrir, de manière exhaustive, l'ensemble des objets exposés (objets matériels ou ceux du patrimoine écrit et graphique). Le statut particulier du pilote sera discuté plus loin.

20 Concernant les modalités de travail, les étudiants sont répartis par groupe de quatre environ. Chaque groupe a accès à une plate-forme collaborative qui rassemble les documents ouverts à tous (cahier des charges et document pilote) et les projets en cours 
de constitution (sous la forme de groupes privés); chacun peut ainsi accéder et contribuer à son projet.

Lorsque les projets sont suffisamment aboutis une brève présentation orale engage à justifier les choix opérés (choix des objets, du phénomène scientifique, type d'activités proposées et organisation du parcours de visite). Cette troisième étape collective incite les membres des autres groupes à questionner les porteurs de projets (informations complémentaires, justification de thèmes, cohérence d'ensemble, niveau de formulation etc.) afin de les inciter à effectuer des remédiations qui contribueront à la clarification des projets.

Enfin, lors d'une dernière étape, chaque groupe teste le guide du visiteur élaboré par un autre groupe sur le lieu d'exposition afin de repérer les points forts et les points faibles du guide. Cette évaluation in situ et entre pairs achève la formation ${ }^{10}$.

\section{2. Objectifs visés pour le dispositif de formation}

Bien que difficiles à séparer étant donné leur imbrication, il est nécessaire, pour les expliciter, de décliner les objectifs généraux attachés à la formation selon deux orientations.

Le premier type concerne ceux qui relèvent de la pédagogie de projet. En référence aux travaux de Perrenoud ${ }^{11}$, la réflexion conduite dans le cadre de la mise en place de la formation prend appui sur les objectifs suivants :

- entrainer la mobilisation de savoirs et de savoir-faire acquis, construire des compétences,

26 - découvrir de nouveaux savoirs dans une perspective de sensibilisation, de «motivation »,

27 - repérer des obstacles et les surmonter au prix de nouveaux apprentissages (dans et hors le projet),

28 - permettre d'identifier des acquis et des manques dans une perspective d'autoévaluation et d'évaluation bilan,

29 - développer la coopération et l'intelligence collective,

30 - renforcer l'identité personnelle et collective,

31 - développer l'autonomie et la capacité de faire des choix et de les négocier.

32 Ces objectifs, qui se déclinent de manière plus ou moins explicite selon chacune des étapes de la formation, renvoient clairement à des dimensions professionnelles du métier d'enseignant ${ }^{12}$; cet aspect sera explicité dans la dernière partie de cette communication.

33 Le deuxième type d'objectifs à identifier, spécifique à la place attribuée à l'EHST dans le dispositif de formation se décline à son tour selon deux orientations distinctes. La première orientation résulte $d u$ choix des objets qu'il convient de documenter en précisant les usages et les pratiques qui leur sont associés.Il s'agit en effet de pouvoir situer les objets dans un réseau scientifique précisant les acteurs (usagers, fabricants, etc. ), les lieux (laboratoire, salle de cours, etc. ) et les savoirs (contenus d'enseignement ou de recherche). Cette approche a pour ambition de permettre l'introduction d'éléments d'histoire des sciences via une histoire sociale et culturelle; elle constitue une première orientation. 

visiteur. La concision des informations, leur hiérarchisation, voire leur reformulation, peut s'avérer nécessaire pour une appropriation par l'enseignant. De fait ce travail de transposition doit tenir compte à la fois des enjeux liés à l'histoire présentée et des contraintes attachées au document lui-même (format et accessibilité). Cette attention portée à la transposition ne se limite pas à l'approche historique, elle concerne chacune des étapes du guide, celui-ci devant dans son ensemble être adapté au public à qui il est destiné.

\section{3. Inscription du dispositif de formation dans l'élaboration du dispositif d'exposition}

Pour appréhender la partie dédiée au dispositif de formation, il paraît essentiel de positionner ce dernier dans un dispositif d'ensemble plus large incluant le processus de conception de l'exposition qui joue un rôle structurant pour la formation.

e premier aspect à souligner concerne l'approche collaborative nécessaire à l'élaboration de l'exposition. Cette conception implique des experts divers tels des conservateurs, archivistes, responsables de collections, médiateurs de culture scientifique, enseignants-chercheurs et des étudiants en médiation et EHST.

Conséquence directe de cette approche, la première étape de conception de l'exposition est marquée par la diversité des préoccupations et des objectifs. Les conservateurs, responsables des collections et archivistes souhaitent «donner à voir " les objets des collections, les exposer, en indiquer la rareté, la provenance ou la valeur (certains objets sont classés au titre des monuments historiques). Les médiateurs souhaitent quant à eux s'emparer des phénomènes scientifiques portés par les objets et les rendre accessibles à tous dans un processus permettant de questionner les phénomènes et de manipuler, tester, expérimenter. Les scientifiques, physiciens et biologistes souhaitent souligner les contenus scientifiques à présenter, leur diversité, leur lien avec des phénomènes naturels et leur actualité. Les chercheurs en EHST visent à contextualiser les objets en indiquant par une biographie de l'objet ${ }^{13}$, les pratiques et usages associés en terme d'enseignement et de recherche. Enfin, c'est à l'expérience esthétique (présentation d'un objet monumental ou d'un texte qui impressionne, d'un dispositif scénique spécifique) que s'attache le scénographe dont les contraintes relatives à un équilibre d'ensemble et à la faisabilité sont également à prendre en compte.

Un objectif partagé réside dans le fait d'engager chaque spectateur dans une rencontre avec l'objet, c'est-à-dire une expérience qui conduise chacun à découvrir l'objet, son fonctionnement, ses usages, ses évolutions, ainsi que les contextes attachés à ces éléments, dans un continu intellectuel et sensible ${ }^{14}$. Les phénomènes scientifiques portés par les objets et les questions de recherche associées font partie de cette contextualisation. Ici le travail de l'historien des sciences joue un rôle essentiel. Il revient à ce dernier de reconstituer à partir des objets et des archives locales (correspondance, notes de cours et de laboratoire, cours manuscrit, publications, catalogue d'inventaire, documents administratifs de la faculté des sciences, compte-rendu de l'Académie des sciences et lettres etc.), les éléments permettant de situer l'objet. A cet égard, le grand télescope grégorien objet emblématique des collections, classé au titre des monuments historiques depuis 2006, peut tenir lieu d'exemple. L'inventaire permet de constater qu'il a été fabriqué par Nairne, alors fabricant d'instruments scientifiques renommé; l'objet 
appartenant à la Société Royale des Sciences est saisi au moment de la révolution française, puis mis en dépôt par la ville à la faculté des sciences. En combinant un intérêt historique qui éclaire la période longue pendant laquelle l'objet est utilisé et un intérêt scientifique qui renseigne les savoirs disciplinaires et leurs évolutions, la biographie de cet objet singulier éclaire, bien au-delà des usages qui lui sont attachés, les réseaux scientifiques qu'il contribue à constituer. L'entrée par la biographie d'objet s'avère particulièrement riche et elle contribue aux choix à effectuer pour le scénario d'ensemble.

Finalement un consensus est obtenu auprès de l'ensemble des acteurs à partir d'un document collaboratif (partagé sur une plate-forme) qualifié de document pilote ou simplement pilote (annexe 1). Conformément aux thèmes généraux choisis pour l'exposition, le document présente les objets et textes illustrés susceptibles d'être exposés, l'ensemble des sujets scientifiques conformes aux programmes scolaires, les expériences et manipulations pouvant être réalisées ainsi qu'un plan du lieu.

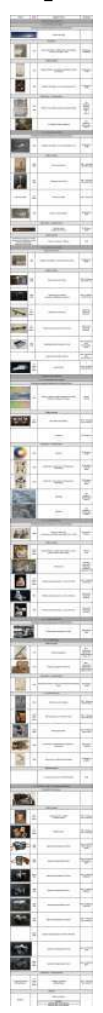

Ce document a une double vocation. Il permet d'une part d'engager une discussion à laquelle chacun peut s'associer compte tenu de sa propre culture et «sans consensus $a$ priori $»^{15}$; il permet d'autre part d'amorcer les négociations nécessaires aux choix qui sont à opérer collectivement et participe à la construction du projet ${ }^{16}$. Dans sa version définitive, le pilote rassemble tous les objets et documents patrimoniaux retenus suite aux discussions et négociations ayant présidé aux différents choix. Ces choix significatifs d'associations cohérentes entre les objets et les documents patrimoniaux, révélateurs d'usages et de pratiques en lien avec l'enseignement et la recherche, constituent une base solide à partir de laquelle des recherches complémentaires (historiques, scientifiques, culturelles etc.) peuvent facilement être conduites.

41 Cette incursion dans le travail de conception de l'exposition, en éclairant le processus d'élaboration du pilote, permet de mieux percevoir le potentiel du document et les 
raisons pour lesquelles il a semblé pertinent de l'intégrer comme référence commune à l'ensemble des projets développés par les étudiants. Devenu un outil pour "penser », le pilote joue ainsi un rôle essentiel dans la formation.

Après cette incursion, un retour sur la formation s'avère nécessaire pour aborder au plus près le travail relatif à la construction des projets et analyser les aspects professionnels qui en résultent.

\section{Objet intermédiaire versus objet frontière et professionnalité}

43 Évaluer le dispositif de formation en fonction des objectifs précisés plus haut ${ }^{17}$ conduit à examiner plus spécifiquement les usages qui ont été faits des documents supports à l'élaboration des projets. Ces éléments permettront ensuite de revenir sur la dimension professionnelle de la formation.

\section{1. Retour d'expérience}

La première étape de la formation pour laquelle des choix (objets, documents patrimoniaux, phénomènes scientifiques) doivent être effectués par les étudiants nécessite examen. Ces choix résultent d'ajustements successifs visant à proposer un parcours conforme au cahier des charges et jugé cohérent dans son ensemble. L'articulation entre la présentation respective des objets, des activités et des phénomènes scientifiques doit également participer de cette cohérence. Il convient en particulier de lier les objets et le phénomène scientifique choisi, afin que ce dernier soit une illustration significative d'une pratique ou d'une notion scientifique attachée à l'objet choisi. Par exemple, en associant les clichés de la Lune ${ }^{18}$ (voir annexe 1) à l'appareil photographique un groupe d'étudiants choisit d'interroger l'évolution des techniques concernant la photographie mais également de travailler sur les phases de la Lune. Pour cet exemple les objets choisis sont des appareils photographiques, de divers époques (fin XIX ${ }^{\mathrm{e}}$ jusqu'au début du XXI ${ }^{e}$ siècle), un extrait du Discours de la méthode de Descartes permettant de découvrir les principes de la réflexion et de la réfraction (nécessaire à la compréhension du fonctionnement de l'appareil photographique) et divers clichés de la Lune, certains datés du milieu du XIXe siècle. C'est le cas également du phénomène de perception des couleurs qui est associé, dans un autre projet, aux modèles anatomiques de Louis Auzoux ${ }^{19}$ (XIX ${ }^{\mathrm{e}}$ siècle), aux planches anatomiques réalisées par Paul Gilis ${ }^{20}$ (XIX ${ }^{\mathrm{e}}$ siècle) et aux cires anatomiques de Félice Fontana (XVIII ${ }^{e}$ siècle) ${ }^{21}$. Puisque chaque modèle, planche ou cire, consiste en une représentation de l'œil humain, le phénomène scientifique de la vision peut alors être introduit à l'aide d'un dispositif expérimental constitué de lentilles diverses. Ainsi, pour chaque sujet d'étude, la constitution d'un corpus légitimé constitue une première étape déterminante.

Si cette étape consacrée au choix des objets nécessite de nombreux ajustements, dont il sera à nouveau question plus loin, les évaluations entre pairs conduisent également à des ajustements. Ainsi, qu'il s'agisse de la présentation orale du projet ou de son évaluation in situ, les projets sont remaniés lors de ces phases afin d'adapter au mieux les propositions à ce qui est supposé permettre un travail avec une classe. Reformulations des textes structurant le parcours, introduction d'un vocabulaire plus adapté et apports 
d'informations constituent ainsi l'essentiel de cette dernière étape d'élaboration du guide du visiteur.

Enfin, ce retour d'expérience doit également inclure les «éléments ludiques » devant figurer dans le guide. Ainsi, les compléments d'informations présentés sous forme de liens url ( $\mathrm{QR} \operatorname{codes}^{22}$ ) et les ouvertures proposées sous forme de jeux (quizz, devinettes, mots croisés etc.) qui achèvent le guide sont traités de manière à réinvestir les éléments historiques et scientifiques du parcours; ils sont généralement jugés bien adaptés lors de l'évaluation in situ.

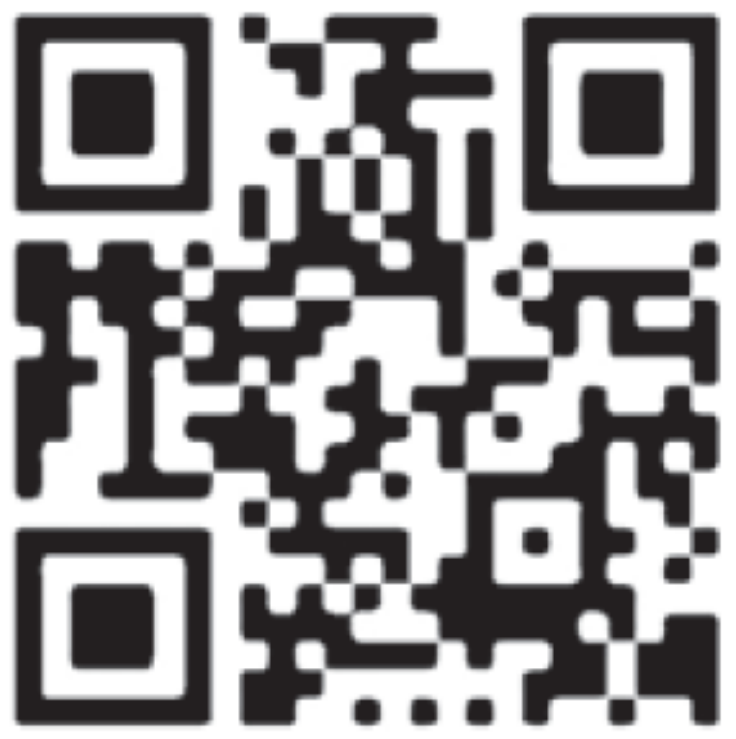

\section{2. Objet intermédiaires versus objet frontière}

Les ajustements et les remaniements évoqués dans la partie précédente constituent des moments clés du dispositif de la formation pour lesquels il s'agit de négocier au sein d'un groupe, défendre un point du vue en argumentant et faire des choix collectivement. Ces actions qui relèvent de compétences professionnelles spécifiques peuvent se développer dans des espaces de réflexions permettant ce travail collaboratif.

Le premier espace repéré concerne l'entrée dans le projet avec la nécessité de faire des choix compte tenu des éléments disponibles pour les étudiants. De fait, le pilote élaboré par les concepteurs de l'exposition, les objets réels et documents patrimoniaux mis en scène sur le lieu d'exposition et le cahier des charges constituent l'ensemble des éléments mis à disposition des étudiants.

Les premiers choix et les premières ébauches du guide du visiteur sont déposés sur une plate-forme dédiée au groupe; cette organisation permet à chaque membre du groupe d'avoir accès à l'ensemble des documents, de pouvoir interagir, faire des suggestions et 
porter des corrections tout en conservant l'historique d'ensemble. Pour le formateur c'est également un moyen de suivre les avancées, les tâtonnements et de prendre part aux discussions si besoin est.

Sans pouvoir généraliser ce processus de conception à l'ensemble des projets, une démarche semble cependant partagée par une majorité de groupes.

51 Ainsi, ce sont les beaux objets, objets monumentaux, objets atypiques présentés sur le lieu d'exposition qui suscitent un véritable attrait ${ }^{23}$ et sont déterminants lors des premiers choix. De fait, le très grand télescope grégorien, le cercle chromatique de Chevreul (XIX siècle) ${ }^{24}$ ou la bulle de savon irisée d'Amadée Guillemin (XIX ${ }^{\mathrm{e}}$ siècle) ${ }^{25}$ remportent l'adhésion du plus grand nombre. De la même manière, les phénomènes scientifiques les plus spectaculaires sont choisis a priori faisant de l'arc en ciel et de l'aurore boréale les phénomènes les plus représentés.

52 Un retour vers le cahier des charges engage à modifier ces premiers choix. Le constat est fait de choix éclectiques, non liés les uns aux autres, de phénomènes scientifiques décontextualisés et de propositions de parcours mobilisant peu, voire pas du tout, les documents mis à disposition. En se recentrant sur la définition de l'objectif principal qui consiste à élaborer un parcours cohérent pour l'enseignant et les élèves, les groupes font émerger les premières questions professionnelles. Quels objectifs un enseignant peut-il fixer lors d'une telle visite, quels types d'activités peut-il proposer, quelle place pour les contenus, quel niveau de formulation avec les élèves en fonction de leur âge? Quelle anticipation peut-il prévoir pour cette visite et quel retour ensuite en classe? Une articulation avec les programmes d'enseignements est-elle envisageable? Quelle documentation doit être fournie?

Compte tenu de ces éléments, les négociations engagées au sein des groupes conduisent à de nouveaux choix. C'est ainsi que l'association des appareils photos et des clichés de Lune ou encore celle des divers modèles de l'œil et du phénomène de perception des couleurs, évoqués plus haut, sont des exemples argumentés significatifs des choix effectués suite aux négociations qui ont animé les groupes. Des retouches successives, pilotées par ces mêmes questions, permettent d'aboutir à une première version du document.

Pour cette étape, les argumentations déployées lors des négociations successives ont pu se déployer en se référant d'une part, au cahier des charges qui définit un cadre et des contraintes, et d'autre part, au document pilote pour les informations qu'il contient.

Dans ce contexte, le document pilote, objet frontière du dispositif de constitution de l'exposition change de statut. De fait, contrairement à l'élaboration du dispositif d'exposition décrit plus haut, les visées attachées au pilote ne sont plus celles relevant d'une diversité de groupes sociaux pour lesquelles il s'agit de s'accorder. En servant de référence, il devient un objet intermédiaire au sens indiqué par Vinck ${ }^{26}, c$ 'est-à-dire un objet qui circule entre les membres d'un réseau en apportant quelque chose à l'action. Reste à souligner le fait que le statut de ce document peut difficilement être discuté indépendamment du cahier des charges et surtout indépendamment des objets et documents patrimoniaux présentés dans l'exposition. L'espace de réflexion ainsi ouvert permet à chacun de se positionner entre des propositions répondant au cadrage strict défini par le cahier des charges, et des propositions conduisant à plus d'autonomie ${ }^{27}$, laissant une place plus grande à l'originalité et l'imagination. C'est à cette condition que les choix peuvent être effectués et l'action conduite. Autrement dit, c'est parce que les 
étudiants se saisissent du document pilote et du cahier des charges pour construire pas à pas leur projet éditorial - le guide du visiteur - que l'action peut s'enclencher. En apportant quelque chose à l'action, en l'accompagnant (rôle de guide) voire en la transformant (incitation à faire de nouveaux choix), le document pilote, auquel doit être associé le cahier des charges, devient un objet intermédiaire. C'est son appropriation par les étudiants, en permettant l'action, qui en fait un objet intermédiaire, c'est-à-dire un document qui ne cesse d'être mobilisé en tant que support à l'action depuis les questionnements initiaux jusqu'à la réalisation du projet.

Les évaluations entre pairs qui engagent également à un retour réflexif au sein des groupes conduisant à de nouveaux ajustements, s'inscrivent dans cette même dynamique. Cette fois encore c'est en référence au document pilote et au cahier des charges que les projets sont critiqués; l'action critique comme les remédiations auxquelles elle engage, résulte de l'objet intermédiaire qui, en jouant le rôle de référence et de guide, sert de catalyseur.

Ainsi, l'analyse critique entre pairs constitue un élément permettant un retour réflexif particulièrement constructif dans la mesure où chacun est informé des objectifs et des modalités d'ensemble. Cette fois encore, les questions soulevées concernent la faisabilité et l'adéquation du parcours à l'enseignant et à la classe ; c'est sur le terrain des questions professionnelles que les remédiations sont discutées.

\section{3. Quid de la professionnalité ?}

Une question centrale réside dans la professionnalité que cette approche permet de développer. Ainsi, c'est en s'adossant aux objets intermédiaires mobilisés dans des espaces de réflexions collaboratifs que la démarche de projet peut se déployer. Elle entraine la mobilisation des savoirs (situer historiquement les objets dans des réseaux) et la construction de compétences (par exemple expérimenter), elle permet d'identifier de nouveaux savoirs (ceux permettant de documenter les objets, ceux en lien avec les phénomènes scientifiques explicités dans les ateliers), ainsi que des acquis et des manques (les évaluations entre pairs jouent ce rôle). Elle développe l'autonomie, la coopération, l'intelligence collective, la capacité à faire des choix et les négocier et elle affirme l'identité personnelle et collective.

En renforçant, dans le cadre d'une approche pluridisciplinaire, la nécessité d'argumenter et de convaincre pour défendre un projet après l'avoir imaginé puis réalisé, le dispositif conçu à partir de l'articulation entre espace de réflexion et objet intermédiaire, permet d'aborder au plus près les objectifs relatifs à la pédagogie de projet.

Par ailleurs il n'est pas nécessaire de spécifier le travail propre à l'histoire des sciences, lorsque celui-ci consiste à adapter au guide du visiteur les éléments collectés pour la contextualisation des objets. En effet, le type d'informations apportées, les niveaux de formulation requis, l'adaptation à la classe etc., renvoient directement aux questions professionnelles évoquées plus haut. Il convient en revanche de spécifier l'apport professionnel relatif au travail d'histoire qui consiste à contextualiser les objets. En travaillant sur la constitution des réseaux qui permettent d'appréhender le processus de structuration et de diffusion des savoirs scientifiques, compte tenu des acteurs et des institutions, c'est à l'exercice d'une pensée critique des disciplines enseignées auquel sont conviés les étudiants. Cette approche réflexive et critique participe également, mais 
autrement, à la formation professionnelle des enseignants qui accèdent par une histoire sociale et culturelle à un point de vue distancié et critique des disciplines enseignées.

61 Avec l'objet frontière, espace de collaboration entre des réseaux très distincts culturellement, et l'objet intermédiaire, espace de collaboration au sein d'un même réseau d'acteurs, l'intérêt réside dans l'action que ces objets permettent en conduisant chacun à une analyse à la fois critique et réflexive interrogeant la professionnalité.

Prétextes théoriques pour conduire cette réflexion, il n'en demeure pas moins que ces objets frontières ou intermédiaires présentent une valeur heuristique incontestable. Si le statut central de l'objet frontière apparaît clairement lors de l'analyse de la conception de l'exposition, celui d'objet intermédiaire n'est pas en reste. Couplé au cahier des charges, le document pilote a joué le rôle de guide (modalités et attentes), de référence (objets et documents accessibles, informations associées), mais aussi de potentiel d'action en présentant de manière organisée des associations d'objets, de documents patrimoniaux et de phénomènes scientifiques pour une thématique donnée. Déclencheur de l'action au sein des espaces de réflexions et activateur de questions professionnelles (incluant les questions d'histoire) l'objet intermédiaire joue, dans la formation, un rôle structurant essentiel.

63 Ici, le dispositif centré sur la pédagogie de projet avec pour objectif la conception et la réalisation d'un document - le guide du visiteur - consiste en une création. Celle-ci n'est cependant possible que dans le cadre d'une réflexion conduite à partir d'un document central, le pilote, qui par sa conception (il est élaboré par des experts) et sa structure (la présentation révèle la cohérence des thèmes, du choix des objets et de leurs associations) constitue une référence. Le projet se construit donc à partir d'allers-retours entre les réflexions permettant d'alimenter la création et les questions nouvelles associées à la création. Finalement cette approche décrite dans l'introduction générale de la revue comme étant un dispositif médian repéré selon la formulation « réfléchir à du créé, pour recréer » $(\mathrm{RCPC})$, permet d'analyser plus finement le dispositif de formation que ne le ferait le dispositif « créer pour réfléchir » $(\mathrm{CPR})$ associé a priori à la pédagogie de projet.

Ces résultats engagent à une réflexion prospective quant aux formations à structurer en lien avec les collections universitaires. Outre les expositions temporaires proposées dans ou hors les murs de l'université et qui devraient pouvoir réinvestir l'expérience constituée autour de l'exposition Regards croisés sur la lumière, une réflexion d'ensemble reste à construire relativement aux collections permanentes qui ne sont pas directement accessibles. Comment en particulier concevoir un document pilote, document intermédiaire dont l'étude montre le rôle central, tant d'un point de vue des informations et des références théoriques qu'il rassemble que de l'action qu'il permet d'engager? A ce stade il semble intéressant d'écrire le scénario "à l'envers" et de supposer que la richesse du document intermédiaire provient de la spécificité attachée à sa conception. Ainsi, dans le cadre de cette hypothèse, l'élaboration de l'objet frontière, obtenu entre experts originaires de domaines disciplinaires divers, constitue une première étape essentielle pour laquelle la structuration spécifique du document résulte du processus de consensus obtenu entre les experts. Ainsi, en partageant préoccupations muséales et perspectives de formations, l'association de conservateurs, responsables de collections, de fonds d'archives patrimoniaux, enseignants chercheurs etc., conduit à l'élaboration d'un pilote qui, en tant qu'objet frontière, s'avère indispensable à la structuration de l'opération muséale tout en constituant la structure de base d'un objet intermédiaire, vecteur de l'action dans le cadre de la formation. 
S'il convient de ne pas limiter les interactions qui jalonnent la formation à la seule appropriation de l'objet intermédiaire et de s'attacher à chaque étape du processus conduisant à la création du guide, il n'en demeure pas moins que la focale portée sur l'objet intermédiaire devrait intéresser la formation professionnelle de manière plus générale.

\section{BIBLIOGRAPHIE}

Caraion, Marta, éd. Usages de l'objet : littérature, histoire, arts et techniques, XIX ${ }^{e}-X^{e}$ siècles. Collection Détours. Seyssel : Champ Vallon, 2014.

Dewey, John, Richard Shusterman, Stewart Buettner, et Jean-Pierre Cometti. L'art comme expérience. Paris : Gallimard, 2010.

Guedj, Muriel. «L'épistémologie et l'histoire des sciences et des techniques peuvent elles aider les futurs enseignants de sciences physiques dans l'exercice de leur métier ? Regards portés pour une ingénierie de formation ». Édité par A. Bernard, M. Dell'Angelo, S. de Montgolfier, A.-S. Godfroy, M. Huchette, A. Mayrargue, et C. Roux. SHS Web of Conferences 13 (2014): 01004. doi $: 10.1051 /$ shsconf/20141301004.

Perrenoud, Philippe. « Apprendre à l'école à travers des projets : pourquoi ? comment ? », 1999. https://www.unige.ch/fapse/SSE/teachers/perrenoud/php_main/php_1999/1999_17.html.

Star, S. L., et. Griesemer J. R. « Institutional Ecology, "Translations" and Boundary Objects: Amateurs and Professionals in Berkeley's Museum of Vertebrate Zoology, 1907-39 ». Social Studies of Science 19, no 3 (1 août 1989): 387-420. Doi :10.1177/030631289019003001.

Perez-Roux, Thérèse. éd., La professionnalité enseignante : modalités de construction en formation, Paideia (Rennes : Presses Univ. de Rennes, 2012).

Vinck, Dominique. «De l'objet intermédiaire à l'objet-frontière : Vers la prise en compte du travail d'équipement ». Revue d'anthropologie des connaissances 3, 1, no 1 (2009) : 51. doi:10.3917/ rac.006.0051.

\section{NOTES}

1. Voir en particulier http://www.shs-conferences.org/articles/shsconf/pdf/2014/10/ shsconf_shst2013_00001.pdf

2. Il s'agit du patrimoine de l'université de Montpellier. Consulter : http://www.umontpellier.fr/ universite/patrimoine/collections/

3. Il s'agit du master MEEF mention premier degré.

4. http://www.umontpellier.fr/wp-content/uploads/2015/01/20151123-Invitation-presse-ExpoRegards-croisés-sur-la-lumière.pdf

5. Désignée Année internationale de la lumière par l'Unesco.

6. Serge Chaumier, «L'objet de musée ?» (Tout garder ? Tout jeter ? Et réinventer ? Dijon : Ville de Dijon, 2010). 
7. Volontairement ici l'expression de guide du visiteur est conservée alors que celle de guide de l'enseignant aurait pu lui être substituée. La précision importante apportée aux parcours laisse finalement une place limitée à une innovation pédagogique et c'est à ce titre que le guide se rapproche d'un guide du visiteur.

8. Le document figure en annexe 2 .

9. Le document figure en annexe 1.

10. Il convient ici de souligner le fait que les guides sont uniquement accessibles aux étudiants de l'UE.

11. Philippe Perrenoud, "Apprendre à l'école à travers des projets : pourquoi ? comment ?", 1999, https://www.unige.ch/fapse/SSE/teachers/perrenoud/php_main/php_1999/1999_17.html. 12. Thérèse Perez-Roux, éd., La professionnalité enseignante: modalités de construction en formation, Paideia (Rennes : Presses Univ. de Rennes, 2012).

13. Alberti, Samuel J. M. « Objects and the Museum ». Isis 96, no 4 (décembre 2005) : 559-71. doi :10.1086/498593.

14. Selon les principes de Dewey, voir : John Dewey et al., L'art comme expérience (Paris : Gallimard, 2010).

15. S. L. Star et J. R. Griesemer, "Institutional Ecology, "Translations" and Boundary Objects: Amateurs and Professionals in Berkeley's Museum of Vertebrate Zoology, 1907-39 ", Social Studies of Science 19, no 3 (1 août 1989) : 387-420, doi:10.1177/030631289019003001.

16. «Espace partagé pour lesquels les objets (communs dans l'espace partagé) sont les ingrédients de l'action ", Ibid.

17. Précisés dans la partie 2.

18. Ceux de Camille Flammarion, voir annexe 1.

19. Louis Auzoux (XIXe siècle) est médecin, il est à l'origine de la création de nombreux modèles anatomiques en papier mâché.

20. Paul Gilis est professeur de médecine à l'université de Montpellier au début du XXe siècle.

21. Felice Fontana est physicien, naturaliste italien du XVIII ${ }^{\mathrm{e}}$ siècle.

22. Générés à partir de logiciels libres.

23. Il ne s'agit pas d'exclure cette dimension qui reste essentielle tout au long du travail. Pour la dimension esthétique voir Marta Caraion, éd., Usages de l'objet : littérature, histoire, arts et techniques, XIX ${ }^{\mathrm{e}}-\mathrm{XX}^{\mathrm{e}}$ siècles, Collection Détours (Seyssel : Champ Vallon, 2014).

24. Michel-Eugene Chevreul est un chimiste français notamment réputé pour la constitution d'un atlas des couleurs très célèbre au XIX ${ }^{\mathrm{e}}$ siècle.

25. Amadée Guillemin est un auteur journaliste français du XIXe siècle passionné de sciences.

26. Dominique Vinck, « De l'objet intermédiaire à l'objet-frontière : Vers la prise en compte du travail d'équipement ", Revue d'anthropologie des connaissances 3, 1, no 1 (2009) : 51, doi $: 10.3917 /$ rac.006.0051.

27. On retrouve ici une problématique pointée par Muriel Guedj qui interroge la place de l'EHST en tant que discipline utilitaire pourvoyant aux problématiques d'autres disciplines ou en tant que discipline permettant de par l'analyse crique à laquelle elle engage d'acquérir une plus grande autonomie. Voir M Guedj « L'épistémologie et l'histoire des sciences et des techniques peuvent-elles aider les futurs enseignants de sciences physiques dans l'exercice de leur métier? Regards portés pour une ingénierie de formation ", éd. par Alain Bernard et al., SHS Web of Conferences 13 (2014) : 01004, doi : 10.1051/shsconf/20141301004. 


\section{RÉSUMÉS}

Collections universitaires et histoire des sciences : étude d'un dispositif pour la formation professionnelle des enseignants. Cette communication analyse un dispositif de formation professionnelle élaboré à partir des collections universitaires pour l'exposition « Regards croisés sur la lumière ». Destinée à de futurs enseignants, la formation vise l'acquisition de compétences professionnelles en associant histoire des sciences et des techniques et éducation scientifique. La présentation et l'analyse de la méthode mise en place pour accompagner le dispositif de formation contribuent à l'explicitation des compétences requises qui engage à une réflexion théorique permettant d'ouvrir de nouvelles perspectives.

This paper analyzes a professional training scheme developed from the university's collections for the exhibition "Regards croisés sur la lumière". Intended for future teachers, the training aims to acquire professional skills by combining history of science and technology and science education. The presentation and analysis of the method put in place to support the training system contribute to the clarification of the required skills, which requires theoretical reflection to open up new perspectives.

\section{INDEX}

Keywords : history of sciences, university collections, Teacher training and education

Mots-clés : histoire de sciences, collections universitaires, formation professionnelle des enseignants

\section{AUTEUR}

\section{MURIEL GUEDJ}

Maître de conférences HDR, Université de Montpellier, LIRDEF 